\title{
Supporting Information for \\ Chiral Lithium Binaphtholate Aqua Complex as a Highly Effective Asymmetric Catalyst for Cyanohydrin Synthesis
}

\author{
Manabu Hatano, Takumi, Ikeno, Takashi, Miyamoto, and Kazuaki Ishihara* \\ Graduate School of Engineering, Nagoya University, \\ Furo-cho, Chikusa, Nagoya 464-8603, Japan
}

General Methods. $\quad{ }^{1} \mathrm{H}$ NMR spectra were measured on a Varian Gemini-2000 spectrometer (300 $\mathrm{MHz}$ ) at ambient temperature. Data were recorded as follows: chemical shift in ppm from internal tetramethylsilane on the TM scale, multiplicity ( $\mathrm{s}=$ singlet; $\mathrm{d}=$ =doublet; $\mathrm{t}=$ triplet; $\mathrm{q}=$ quartet; $\mathrm{m}=$ multiplet), coupling constant $(\mathrm{Hz})$, integration, and assignment. ${ }^{13} \mathrm{C} \mathrm{NMR}$ spectra were measured on Varian Gemini-2000 (75 MHz) spectrometer. Chemical shifts were recorded in ppm from the solvent resonance employed as the internal standard (deuterochloroform at $77.10 \mathrm{ppm}$ ). Low-resolution mass analysis (LRMS) and GC analysis were performed with a Shimadzu GC/MS instrument [GC-17A/QP-5050A; column: TC-1 $(0.25 \mathrm{~mm} \times 30 \mathrm{~m})$ ] by direct insertion for chemical ionization (CI) with isobutane. High performance liquid chromatography (HPLC) analysis was conducted using Shimadzu LC-10 AD coupled diode array-detector SPD-MA-10A-VP and chiral column of Daicel CHIRALCEL, CHIRALPAK; OD-H (4.6 mm × $25 \mathrm{~cm})$, OJ-H $(4.6 \mathrm{~mm} \times 25 \mathrm{~cm})$, AD-H $(4.6 \mathrm{~mm} \times 25 \mathrm{~cm})$, AS-H $(4.6 \mathrm{~mm} \times 25 \mathrm{~cm})$. GC analysis was performed with Shimadzu 17A instruments using CP-Cyclodextrin- $\beta$-2,3,6-M-19 (i.d. $0.25 \mathrm{~mm} \times 25 \mathrm{~m}$; CHROMPACK; GL Science Inc.) or CHIRALDEX B-DM (i.d. 0.25 mm $\times 20$ m; Tokyo Kasei Kogyo Co., LTD). All experiments were carried out under an atmosphere of dry nitrogen. For thin-layer chromatography (TLC) analysis throughout this work, Merck precoated TLC plates (silica gel 60GF254 $0.25 \mathrm{~mm}$ ) were used. The products were purified by neutral column chromatography on silica gel (Kanto Chemical Co., Inc. 37560). Visualization was accomplished by UV light (254 $\mathrm{nm}$ ), anisaldehyde, $\mathrm{KMnO}_{4}$ and phosphomolybdic acid. In experiments that required dry solvents, diethyl ether (dehydrate), toluene (dehydrate), dichloromethane (dehydrate), and tetrahydrofuran (dehydrate) were purchased from Kanto Chemical Co., Inc. $n$-BuLi (1.56 $M$ in hexane, Kanto Chemical Co., Inc.), $\mathrm{LiOH}$ (Merck), $\mathrm{LiOH} \cdot \mathrm{H}_{2} \mathrm{O}$ (Wako), $\mathrm{LiOPr}^{i}$ (Alfa Aesar), $\mathrm{LiOAc}$ (Acros), $\mathrm{Li}_{2} \mathrm{O}$ (Strem), LiOMe (Aldrich), LiOEt (Aldrich).

\section{Representative Procedure for Catalytic Asymmetric Cyanation with $(R)$-BINOL} /n-BuLi (Table 1). To a stirred solution of (R)-BINOL (28.6 mg, $0.10 \mathrm{mmol})$ in $2 \mathrm{~mL}$ of toluene was added dropwise $n$-BuLi (1.56 $\mathrm{M}$ in hexane, $64.1 \mu \mathrm{L}, 0.10 \mathrm{mmol})$ at room temperature under nitrogen atmosphere, and the mixture was stirred for $10 \mathrm{~min}$. Benzaldehyde (101.6 $\mu \mathrm{L}, 1.0 \mathrm{mmol}$ ) was added and the mixture was stirred for $20 \mathrm{~min}$ at room temperature. This yellow solution was 
cooled to $-78{ }^{\circ} \mathrm{C}$ and stirred for $10 \mathrm{~min}$ at that temperature. Trimethylsilylcyanide [Caution!] (133.1 $\mu \mathrm{L}, 1.0 \mathrm{mmol}$ ) was added dropwise, and after stirring for $1 \mathrm{~h}$ the reaction mixture was diluted with $10 \mathrm{w} / \mathrm{w} \% \mathrm{HCl} / \mathrm{MeOH}(1 \mathrm{~mL})$ at $-78{ }^{\circ} \mathrm{C}$. After $10 \mathrm{~min}$, water $(10 \mathrm{~mL})$ and ethyl acetate $(10 \mathrm{~mL})$ were added. The product was extracted with ethyl acetate $(10 \mathrm{~mL} \times 2)$ and washed by brine $(10 \mathrm{~mL})$, dried over $\mathrm{MgSO}_{4}$ and evaporated under reduced pressure to give the cyanohydrin. To a solution of the crude cyanohydrin in dichloromethane $(2 \mathrm{~mL})$ were added pyridine $(0.16 \mathrm{~mL}, 2 \mathrm{mmol})$ and acetic anhydride $(0.14 \mathrm{~mL}, 1.5 \mathrm{mmol})$ at $-78{ }^{\circ} \mathrm{C}$. The mixture was stirred at room temperature for $30 \mathrm{~min}$, diluted with ethyl acetate (20 mL) and $1 \mathrm{~N} \mathrm{HCl}(10$ $\mathrm{mL})$. The organic phase was separated, washed with water $(10 \mathrm{~mL})$, sat. $\mathrm{NaHCO}_{3}$ solution $(10$ $\mathrm{mL})$, and brine $(10 \mathrm{~mL})$, dried over $\mathrm{MgSO}_{4}$ and evaporated under reduced pressure to give the cyanohydrin acetate which was purified by neutral silica gel column chromatography (hexane/AcOEt $=10 / 1)$ giving the desired products as a colorless oil ( $>99 \%$ yield).

\section{Representative Procedure for Catalytic Asymmetric Cyanation with $(R)$-BINOL} $/ \mathbf{n}$-BuLi// $\mathbf{H}_{2} \mathbf{O}$ (Table 1). To a stirred solution of $(R)$-BINOL (28.6 mg, $0.10 \mathrm{mmol}$ ) in $2 \mathrm{~mL}$ of wet-toluene (including $0.030 \mathrm{mmol} \mathrm{H}_{2} \mathrm{O}$ per $2 \mathrm{~mL}$ of toluene) was added dropwise $n$-BuLi (1.56 M in hexane, $64.1 \mu \mathrm{L}, 0.10 \mathrm{mmol}$ ) at room temperature under nitrogen atmosphere, and the mixture was stirred for $10 \mathrm{~min}$. Benzaldehyde $(101.6 \mu \mathrm{L}, 1.0 \mathrm{mmol})$ was added and the mixture was stirred for $20 \mathrm{~min}$ at room temperature. This yellow solution was cooled to $-78^{\circ} \mathrm{C}$ and stirred for $10 \mathrm{~min}$ at that temperature. Trimethylsilylcyanide [Caution!] (133.1 $\mu \mathrm{L}, 1.0 \mathrm{mmol})$ was added dropwise, and after stirring for $1 \mathrm{~h}$ the reaction mixture was diluted with $10 \mathrm{w} / \mathrm{w} \% \mathrm{HCl} / \mathrm{MeOH}$ (1 $\mathrm{mL})$ at $-78{ }^{\circ} \mathrm{C}$. After $10 \mathrm{~min}$, water $(10 \mathrm{~mL})$ and ethyl acetate $(10 \mathrm{~mL})$ were added. The product was extracted with ethyl acetate $(10 \mathrm{~mL} \times 2)$ and washed by brine $(10 \mathrm{~mL})$, dried over $\mathrm{MgSO}_{4}$ and evaporated under reduced pressure to give the cyanohydrin. (Method A): To a solution of the crude cyanohydrin in dichloromethane $(2 \mathrm{~mL})$ were added pyridine $(0.16 \mathrm{~mL}, 2$ mmol) and acetic anhydride $(0.14 \mathrm{~mL}, 1.5 \mathrm{mmol})$ at $-78{ }^{\circ} \mathrm{C}$. The mixture was stirred at room temperature for $30 \mathrm{~min}$, diluted with ethyl acetate $(20 \mathrm{~mL})$ and $1 N \mathrm{HCl}(10 \mathrm{~mL})$. The organic phase was separated, washed with water $(10 \mathrm{~mL})$, sat. $\mathrm{NaHCO}_{3}$ solution $(10 \mathrm{~mL})$, and brine $(10$ $\mathrm{mL}$ ), dried over $\mathrm{MgSO}_{4}$ and evaporated under reduced pressure to give the cyanohydrin acetate which was purified by neutral silica gel column chromatography (hexane/AcOEt $=10 / 1$ ) giving the desired products as a colorless oil (95\% yield).

Representative Procedure for Catalytic Asymmetric Cyanation with 10 mol\% of (R)-BINOL/LiOPr ${ }^{i}$ (Table 2). The mixture of $(R)$-BINOL (28.6 mg, $0.10 \mathrm{mmol}$ ) and LiOPr ${ }^{i}(6.6$ 
$\mathrm{mg}, 0.1 \mathrm{mmol})$ in toluene $(2 \mathrm{~mL})$ was stirred at room temperature for 20 min under nitrogen atmosphere. Benzaldehyde (101.6 $\mu \mathrm{L}, 1.0 \mathrm{mmol})$ was added and the mixture was stirred for 20 min at room temperature. This pale yellow solution was cooled to $-78{ }^{\circ} \mathrm{C}$ and stirred for $10 \mathrm{~min}$ at that temperature. Trimethylsilylcyanide [Caution!] (133.1 $\mu \mathrm{L}, 1.0 \mathrm{mmol})$ was added dropwise, and after stirring for $1 \mathrm{~h}$ the reaction mixture was diluted with $10 \mathrm{w} / \mathrm{w} \% \mathrm{HCl} / \mathrm{MeOH}(1 \mathrm{~mL})$ at $78{ }^{\circ} \mathrm{C}$. After $10 \mathrm{~min}$, water $(10 \mathrm{~mL})$ and ethyl acetate $(10 \mathrm{~mL})$ were added. The product was extracted with ethyl acetate $(10 \mathrm{~mL} \times 2)$ and washed by brine $(10 \mathrm{~mL})$, dried over $\mathrm{MgSO}_{4}$ and evaporated under reduced pressure to give the cyanohydrin. $\left[{ }^{1} \mathrm{H}\right.$ NMR $\left(300 \mathrm{MHz}, \mathrm{CDCl}_{3}\right) \delta 3.21(\mathrm{~d}$, $J=6.3 \mathrm{~Hz}, 1 \mathrm{H}), 5.52$ (d, $J=6.3 \mathrm{~Hz}, 1 \mathrm{H}), 7.40-7.60(5 \mathrm{H}) .{ }^{13} \mathrm{C}$ NMR $\left(75 \mathrm{MHz}, \mathrm{CDCl}_{3}\right) \delta$ 63.7, 118.8, 126.7, 129.2, 129.9, 135.2.] (95\% ee $(S),[\alpha]_{\mathrm{D}}{ }^{22}-42.2$ (c 0.91, $\left.\mathrm{CHCl}_{3}\right)$; Lit. $^{3}[\alpha]_{\mathrm{D}}{ }^{20}-46.5$ (c 1.4, $\mathrm{CHCl}_{3}$ ) for $>99 \%$ ee $(S)$ ). (Method A): To a solution of the crude cyanohydrin in dichloromethane $(2 \mathrm{~mL})$ were added pyridine $(0.16 \mathrm{~mL}, 2 \mathrm{mmol})$ and acetic anhydride $(0.14 \mathrm{~mL}$, $1.5 \mathrm{mmol}$ ) at $-78^{\circ} \mathrm{C}$. The mixture was stirred at room temperature for $30 \mathrm{~min}$, diluted with ethyl acetate $(20 \mathrm{~mL})$ and $1 \mathrm{~N} \mathrm{HCl}(10 \mathrm{~mL})$. The organic phase was separated, washed with water (10 $\mathrm{mL})$, sat. $\mathrm{NaHCO}_{3}$ solution $(10 \mathrm{~mL})$, and brine $(10 \mathrm{~mL})$, dried over $\mathrm{MgSO}_{4}$ and evaporated under reduced pressure to give the cyanohydrin acetate which was purified by neutral silica gel column chromatography (hexane/AcOEt $=10 / 1)$ giving the desired products as a colorless oil (175.2 mg, $>99 \%$ yield). (Method B): To a solution of the crude cyanohydrin in acetonitrile (2 mL) were added scandium trifluoromethanesulfonate $(4.9 \mathrm{mg}, 0.01 \mathrm{mmol})$ and acetic anhydride $(0.14 \mathrm{~mL}, 1.5$ $\mathrm{mmol}$ ) at $-78{ }^{\circ} \mathrm{C}$. The mixture was stirred at room temperature for $30 \mathrm{~min}$, then evaporated under reduced pressure to give the cyanohydrin acetate which was purified by neutral silica gel column chromatography (hexane/AcOEt $=10 / 1)$ giving the desired products as a colorless oil $(175.2 \mathrm{mg}$, 95\% yield). Method B was exclusively adopted for the corresponding cyanohydrins of $\mathbf{3 h}$ and $\mathbf{3 i}$. In both method A and B, the enantiomeric purity was determined by chiral GC $\left[120{ }^{\circ} \mathrm{C}, \mathrm{t}_{\mathrm{R}}(R)=\right.$ 23.8 min, $\mathrm{t}_{\mathrm{R}}(S)=27.3$ min (major)] $\left(95 \%\right.$ ee $(S),[\alpha]_{\mathrm{D}}{ }^{21}-6.92$ (c 2.20, $\left.\mathrm{CHCl}_{3}\right)$; Lit. $^{3}[\alpha]_{\mathrm{D}}{ }^{20}-7.24$ (c $2.3, \mathrm{CHCl}_{3}$ ) for $>99 \%$ ee $(S)$ ).

\section{Representative Procedure for Catalytic Asymmetric Cyanation with 1 mol\% of} (R)-BINOL/LiOPr ${ }^{i}$ (Table 2). The mixture of (R)-BINOL (28.6 mg, $0.10 \mathrm{mmol}$ ) and $\operatorname{LiOPr}^{i}$ (6.6 $\mathrm{mg}, 0.1 \mathrm{mmol})$ in toluene $(2 \mathrm{~mL})$ was stirred at room temperature for $20 \mathrm{~min}$ under nitrogen atmosphere. Benzaldehyde $(1.016 \mathrm{~mL}, 10.0 \mathrm{mmol})$ was added and the mixture was stirred for 20 min at room temperature. This pale yellow solution was cooled to $-78{ }^{\circ} \mathrm{C}$ and stirred for $10 \mathrm{~min}$ at that temperature. Trimethylsilylcyanide [Caution!] (1.331 mL, $10.0 \mathrm{mmol})$ was added dropwise, and after stirring for $1 \mathrm{~h}$ the reaction mixture was diluted with $10 \mathrm{w} / \mathrm{w} \% \mathrm{HCl} / \mathrm{MeOH}(5 \mathrm{~mL})$ at - 
$78{ }^{\circ} \mathrm{C}$. After $10 \mathrm{~min}$, water $(10 \mathrm{~mL})$ and ethyl acetate $(10 \mathrm{~mL})$ were added. The product was extracted with ethyl acetate $(20 \mathrm{~mL} \times 3)$ and washed by brine $(30 \mathrm{~mL})$, dried over $\mathrm{MgSO}_{4}$ and evaporated under reduced pressure to give the cyanohydrin. To a solution of the crude cyanohydrin in dichloromethane $(2 \mathrm{~mL})$ were added pyridine $(1.6 \mathrm{~mL}, 20 \mathrm{mmol})$ and acetic anhydride $(1.4 \mathrm{~mL}, 15 \mathrm{mmol})$ at $-78^{\circ} \mathrm{C}$. The mixture was stirred at room temperature for $30 \mathrm{~min}$, diluted with ethyl acetate (30 mL) and $1 \mathrm{~N} \mathrm{HCl}(30 \mathrm{~mL})$. The organic phase was separated, washed with water $(30 \mathrm{~mL})$, sat. $\mathrm{NaHCO}_{3}$ solution $(30 \mathrm{~mL})$, and brine $(30 \mathrm{~mL})$, dried over $\mathrm{MgSO}_{4}$ and evaporated under reduced pressure to give the cyanohydrin acetate which was purified by neutral silica gel column chromatography (hexane/AcOEt $=10 / 1$ ) giving the desired products as a colorless oil (1.716 g, 98\% yield).

Cyano(phenyl)methyl acetate (Derived from 4a) ${ }^{1,2,3,4,5}$

${ }^{1} \mathrm{H}$ NMR (300 MHz, $\mathrm{CDCl}_{3}$ ) $\delta 2.16$ (s, 3H), 6.43 (s, 1H), 7.40-7.60 (m, 5H). ${ }^{13} \mathrm{C} \mathrm{NMR}\left(75 \mathrm{MHz}, \mathrm{CDCl}_{3}\right) \delta 20.5$, 62.8, 116.1, 127.8, 129.2, 130.4, 131.7, 168.9. Chiral GC CP-Cyclodextrin- $\beta-2,3,6-\mathrm{M}-19\left[120{ }^{\circ} \mathrm{C}, \mathrm{t}_{\mathrm{R}}(R)=23.8\right.$ $\left.\min , \mathrm{t}_{\mathrm{R}}(S)=27.3 \mathrm{~min}\right] . \quad[\alpha]_{\mathrm{D}}{ }^{21}-6.92\left(\right.$ c 2.20, $\left.\mathrm{CHCl}_{3}\right)$ for $95 \%$ ee of $(S)-4 \mathbf{a}$; $\mathrm{Lit}^{3}{ }^{3}[\alpha]_{\mathrm{D}}{ }^{20}-7.24\left(\right.$ c 2.3, $\left.\mathrm{CHCl}_{3}\right)$ for $>99 \%$ ee $(S))$.

Cyano(4-fluorophenyl)methyl acetate (Derived from $\mathbf{4 b})^{2,5}$

${ }^{1} \mathrm{H}$ NMR (300 MHz, CDCl 3 ) $\delta 2.18$ (s, 3H), 6.39 (s, 1H), 7.44 (dd, $J=9.0,8.4 \mathrm{~Hz}, 2 \mathrm{H}$ ), 7.44 (dd, $J=9.0,5.1 \mathrm{~Hz}$, 2H). ${ }^{13}$ C NMR (75 MHz, $\mathrm{CDCl}_{3}$ ) $\delta$ 20.2, 62.0, 115.8, 116.2 (d, $J=21.7 \mathrm{~Hz}$ ), 127.7 (d, $J=3.5 \mathrm{~Hz}$ ), 9.15 (d $J=9.2$ $\mathrm{Hz}), 163.6(\mathrm{~d}, J=249.5 \mathrm{~Hz}), 168.7 .{ }^{19} \mathrm{~F} \mathrm{NMR}\left(282 \mathrm{MHz}, \mathrm{CDCl}_{3}\right) \delta-110.24$ (s, 1F). Chiral GC CP-Cyclodextrin- $\beta-2,3,6-\mathrm{M}-19\left[150^{\circ} \mathrm{C}, \mathrm{t}_{\mathrm{R}}(R)=7.9 \mathrm{~min}, \mathrm{t}_{\mathrm{R}}(S)=8.5 \mathrm{~min}\right]$.

Cyano(3-fluorophenyl)methyl acetate (Derived from 4c) ${ }^{2}$ ${ }^{1} \mathrm{H}$ NMR (300 MHz, CDCl 3 ) $\delta 2.19$ (s, 3H), 6.40 (s, 1H), 7.15 (m, 1H), 7.24 (m, 1H), 7.31 (m, 1H), 7.44 (m, 1H). ${ }^{13} \mathrm{C}$ NMR $\left(75 \mathrm{MHz}, \mathrm{CDCl}_{3}\right.$ ) $\delta$ 20.1, 61.9, 114.7 (d, $\left.J=22.8 \mathrm{~Hz}\right), 115.5,117.2$ (d, $\left.J=20.6 \mathrm{~Hz}\right), 123.3$ (d, $J=3.5$ $\mathrm{Hz}), 130.9$ (d, $J=8.0 \mathrm{~Hz}), 133.8$ (d, $J=6.9 \mathrm{~Hz}), 162.6$ (d, $J=247.2 \mathrm{~Hz}), 168.6 .{ }^{19} \mathrm{~F}$ NMR $\left(282 \mathrm{MHz}, \mathrm{CDCl}_{3}\right)$ $\delta$-111.31 (s, 1F). Chiral GC CP-Cyclodextrin- $\beta-2,3,6-\mathrm{M}-19\left[140{ }^{\circ} \mathrm{C}, \mathrm{t}_{\mathrm{R}}(R)=10.5 \mathrm{~min}, \mathrm{t}_{\mathrm{R}}(S)=11.5 \mathrm{~min}\right]$.

(4-Chlorophenyl)(cyano)methyl acetate (Derived from 4d) ),2,4 $^{1,4}$

${ }^{1} \mathrm{H}$ NMR (300 MHz, CDCl $) \delta 2.17$ (s, 3H), 6.38 (s, 1H), 7.41-7.54 (m, 4H). ${ }^{13} \mathrm{C}$ NMR $\left(75 \mathrm{MHz}, \mathrm{CDCl}_{3}\right) \delta 20.3$, 62.1, 115.7, 129.2, 129.4, 130.2, 136.5, 168.7. Chiral GC CP-Cyclodextrin- $\beta-2,3,6-\mathrm{M}-19\left[150{ }^{\circ} \mathrm{C}, \mathrm{t}_{\mathrm{R}}(R)=18.4\right.$ $\left.\min , \mathrm{t}_{\mathrm{R}}(S)=19.9 \mathrm{~min}\right]$. 
(3-Chlorophenyl)(cyano)methyl acetate (Derived from 4e) (2,4 $^{2,}$

${ }^{1} \mathrm{H}$ NMR (300 MHz, CDCl $) \delta 2.19$ (s, 3H), 6.40 (s, 1H), 7.38-7.64 (m, 4H). $\left.{ }^{13} \mathrm{C} \mathrm{NMR} \mathrm{(75} \mathrm{MHz,} \mathrm{CDCl}_{3}\right) \delta 20.3$, 62.0, 115.5, 125.8, 127.9, 130.5, 130.6, 133.5, 135.2, 168.6. Chiral GC CP-Cyclodextrin- $\beta-2,3,6-\mathrm{M}-19\left[140{ }^{\circ} \mathrm{C}\right.$, $\left.\mathrm{t}_{\mathrm{R}}(R)=16.9 \min , \mathrm{t}_{\mathrm{R}}(S)=17.9 \mathrm{~min}\right]$.

(4-Bromophenyl)(cyano)methyl acetate (Derived from $\mathbf{4 f})^{2}$

${ }^{1} \mathrm{H}$ NMR (300 MHz, CDCl $) \delta 2.17$ (s, 3H), 6.37 (s, 1H), 7.40 (q, $\left.J=8.4 \mathrm{~Hz}, 2 \mathrm{H}\right), 7.60$ (q, $\left.J=8.7 \mathrm{~Hz}, 2 \mathrm{H}\right) .{ }^{13} \mathrm{C}$ NMR $\left(75 \mathrm{MHz}, \mathrm{CDCl}_{3}\right) \delta 20.3,62.1,115.6,124.7,129.4,130.7,132.4$, 168.7. Chiral GC CP-Cyclodextrin- $\beta-2,3,6-\mathrm{M}-19\left[160{ }^{\circ} \mathrm{C}, \mathrm{t}_{\mathrm{R}}(R)=19.6 \mathrm{~min}, \mathrm{t}_{\mathrm{R}}(S)=20.7 \mathrm{~min}\right]$.

(3-Bromophenyl)(cyano)methyl acetate (Derived from $\mathbf{4 g})^{2}$

${ }^{1} \mathrm{H}$ NMR (300 MHz, CDCl $) \delta 2.19$ (s, 3H), 6.37 (s, 1H), 7.34 (t, $\left.J=8.1 \mathrm{~Hz}, 1 \mathrm{H}\right), 7.47$ (d, $\left.J=7.8 \mathrm{~Hz}, 1 \mathrm{H}\right), 7.60$ (d, $J=7.8 \mathrm{~Hz}, 1 \mathrm{H}), 7.67$ (bs, $1 \mathrm{H}) .{ }^{13} \mathrm{C}$ NMR $\left(75 \mathrm{MHz}, \mathrm{CDCl}_{3}\right) \delta 20.3,61.9,115.5,123.1,126.3,130.7,130.7$, 133.5, 133.7, 168.6. Chiral GC CP-Cyclodextrin- $\beta-2,3,6-\mathrm{M}-19\left[150{ }^{\circ} \mathrm{C}, \mathrm{t}_{\mathrm{R}}(R)=26.1 \mathrm{~min}, \mathrm{t}_{\mathrm{R}}(S)=27.7 \mathrm{~min}\right]$.

Cyano[4-(trifluoromethyl)phenyl]methyl acetate (Derived from $\mathbf{4 h})^{1,4}$ ${ }^{1} \mathrm{H}$ NMR (300 MHz, CDCl 3 ) $\delta 2.20$ (s, 3H), 6.47 (s, 1H), 7.67 (t, $\left.J=8.4 \mathrm{~Hz}, 2 \mathrm{H}\right), 7.74(\mathrm{~d}, J=8.4 \mathrm{~Hz}, 2 \mathrm{H}) .{ }^{13} \mathrm{C}$ NMR (75 MHz, $\left.\mathrm{CDCl}_{3}\right) \delta$ 20.1, 62.0, 115.4, 123.4 (q, $\left.J=270.0 \mathrm{~Hz}\right), 126.1$ (q, $\left.J=3.5 \mathrm{~Hz}\right), 128.1,132.3(\mathrm{q}, J=$

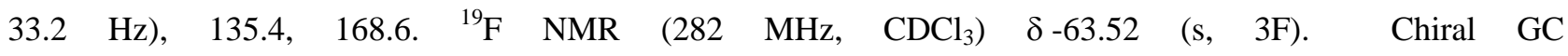
CP-Cyclodextrin- $\beta-2,3,6-\mathrm{M}-19\left[140{ }^{\circ} \mathrm{C}, \mathrm{t}_{\mathrm{R}}(R)=11.4 \mathrm{~min}, \mathrm{t}_{\mathrm{R}}(S)=12.8 \mathrm{~min}\right]$.

Cyano[3-(trifluoromethyl)phenyl]methyl acetate (Derived from $\mathbf{4 i})^{6}$ ${ }^{1} \mathrm{H}$ NMR (300 MHz, CDCl $) \delta 2.20$ (s, 3H), 6.47 (s, 1H), 7.61 (t, $\left.J=7.5 \mathrm{~Hz}, 1 \mathrm{H}\right), 7.70-7.81(\mathrm{~m}, 3 \mathrm{H}) .{ }^{13} \mathrm{C}$ NMR $\left(75 \mathrm{MHz}, \mathrm{CDCl}_{3}\right) \delta$ 20.3, 62.1, 115.5, 123.5 (q, $\left.J=270.1 \mathrm{~Hz}\right), 124.6$ (q, $\left.J=3.5 \mathrm{~Hz}\right), 127.3$ (q, $\left.J=3.5 \mathrm{~Hz}\right), 130.0$, 131.2, 131.8 (q, $J=33.2 \mathrm{~Hz}), 132.8,168.8 .{ }^{19} \mathrm{~F}$ NMR $\left(282 \mathrm{MHz}, \mathrm{CDCl}_{3}\right) \delta-63.34(\mathrm{~s}, 3 \mathrm{~F}) . \quad$ Chiral GC CP-Cyclodextrin- $\beta-2,3,6-\mathrm{M}-19\left[130{ }^{\circ} \mathrm{C}, \mathrm{t}_{\mathrm{R}}(R)=13.4 \mathrm{~min}, \mathrm{t}_{\mathrm{R}}(S)=16.1 \mathrm{~min}\right]$.

Cyano( $m$-tolyl)methyl acetate (Derived from $\mathbf{4 j})^{1,4}$

${ }^{1} \mathrm{H}$ NMR (300 MHz, CDCl 3 ) $\delta 2.17$ (s, 3H), 2.40 (s, 3H), 6.37 (s, 1H), 7.20-7.45 (m, 4H). ${ }^{13} \mathrm{C}$ NMR (75 MHz, $\left.\mathrm{CDCl}_{3}\right) \delta$ 20.3, 21.2, 62.8, 116.2, 124.9, 128.4, 129.0, 131.1, 131.6, 139.1, 168.8. Chiral GC CP-Cyclodextrin- $\beta-2,3,6-\mathrm{M}-19\left[150{ }^{\circ} \mathrm{C}, \mathrm{t}_{\mathrm{R}}(R)=11.0 \mathrm{~min}, \mathrm{t}_{\mathrm{R}}(S)=11.5 \mathrm{~min}\right]$.

Cyano(3-methoxyphenyl)methyl acetate (Derived from $\mathbf{4 k})^{1,2,3,4,5}$

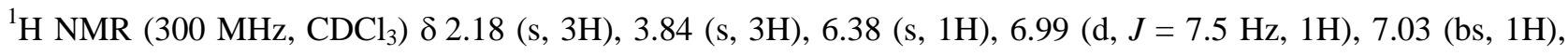


7.09 (d, $J=7.5 \mathrm{~Hz}, 1 \mathrm{H}), 7.36(\mathrm{t}, J=8.1 \mathrm{~Hz}, 1 \mathrm{H}) .{ }^{13} \mathrm{C} \mathrm{NMR}\left(75 \mathrm{MHz}, \mathrm{CDCl}_{3}\right) \delta$ 20.4, 55.3, 62.6, 113.2, 115.9, 116.0, 119.9, 130.3, 133.0, 160.0, 168.8. Chiral GC CP-Cyclodextrin- $\beta-2,3,6-\mathrm{M}-19\left[150{ }^{\circ} \mathrm{C}, \mathrm{t}_{\mathrm{R}}(R)=22.1 \mathrm{~min}\right.$, $\left.\mathrm{t}_{\mathrm{R}}(S)=23.4 \mathrm{~min}\right]$.

Cyano(3,5-dimethoxyphenyl)methyl acetate (Derived from $\mathbf{4 l})^{2}$

${ }^{1} \mathrm{H}$ NMR (300 MHz, CDCl ${ }_{3}$ ) $\delta 2.18$ (s, 3H), 3.82 (s, 6H), 6.32 (s, 1H), 6.51 (s, 2H), 6.34 (s, 2H). ${ }^{13} \mathrm{C}$ NMR (75 $\left.\mathrm{MHz}, \mathrm{CDCl}_{3}\right) \delta 20.4,55.5,62.7,102.1,105.7,116.0,133.6,161.3,168.8$. Chiral GC CHIRALDEX B-DM $\left[160{ }^{\circ} \mathrm{C}, \mathrm{t}_{\mathrm{R}}(R)=10.3 \mathrm{~min}, \mathrm{t}_{\mathrm{R}}(S)=11.2 \mathrm{~min}\right]$.

Cyano( $\alpha$-naphthyl)methyl acetate (Derived from $\mathbf{4 m})^{2,4}$

${ }^{1} \mathrm{H}$ NMR (300 MHz, CDCl $)$ ) 2.22 (s, 3H), 7.03 (s, 1H), 7.49-7.70 (m, 3H), 7.82 (d, $J=7.2 \mathrm{~Hz}, 1 \mathrm{H}$ ), 7.92-8.01 (m, 2H), 8.02 (d, $J=8.4 \mathrm{~Hz}, 1 \mathrm{H}) .{ }^{13} \mathrm{C}$ NMR $\left(75 \mathrm{MHz}, \mathrm{CDCl}_{3}\right) \delta 20.2,61.1,116.0,122.4,124.9,126.4,126.8$, 127.4, 127.5, 129.0, 129.9, 131.3, 133.7, 168.9. Chiral HPLC $(\mathrm{OD}-\mathrm{H}$; hexane/IPA $=20 / 1,0.5 \mathrm{~mL} / \mathrm{min})\left[\mathrm{t}_{\mathrm{R}}(R)=\right.$ $\left.25.52 \mathrm{~min}, \mathrm{t}_{\mathrm{R}}(S)=30.37 \mathrm{~min}\right]$.

Cyano( $\beta$-naphthyl)methyl acetate (Derived from 4n) ${ }^{1,2,4}$

${ }^{1} \mathrm{H}$ NMR (300 MHz, $\mathrm{CDCl}_{3}$ ) $\delta 2.19$ (s, 3H), 6.58 (s, 1H), 6.95 (d, $\left.J=8.7 \mathrm{~Hz}, 2 \mathrm{H}\right), 7.45(\mathrm{~d}, J=8.7 \mathrm{~Hz}, 2 \mathrm{H}) .{ }^{13} \mathrm{C}$ NMR (75 MHz, $\left.\mathrm{CDCl}_{3}\right) \delta$ 20.2, 62.8, 116.0, 124.0, 126.8, 127.3, 127.6, 127.7, 128.1, 128.7, 129.2, 132.6, 133.6, 168.7. Chiral GC CP-Cyclodextrin- $\beta-2,3,6-\mathrm{M}-19\left[160^{\circ} \mathrm{C}, \mathrm{t}_{\mathrm{R}}(R)=40.1 \mathrm{~min}, \mathrm{t}_{\mathrm{R}}(S)=40.9 \mathrm{~min}\right]$.

Cyano(3-furanyl)methyl acetate (Derived from 4o) ${ }^{3}$

${ }^{1} \mathrm{H}$ NMR $\left(300 \mathrm{MHz}, \mathrm{CDCl}_{3}\right.$ ) $\delta 2.16$ (s, 3H), 6.37 (s, 1H), 6.54 (s, 1H), 7.49 (s, 1H), 7.68 (s, 1H). ${ }^{13} \mathrm{C}$ NMR $(75$ $\left.\mathrm{MHz}, \mathrm{CDCl}_{3}\right) \delta$ 20.3, 55.4, 109.1, 115.5, 117.9, 142.4, 144.4, 168.8. Chiral GC CP-Cyclodextrin- $\beta-2,3,6-\mathrm{M}-19$ $\left[120^{\circ} \mathrm{C}, \mathrm{t}_{\mathrm{R}}(R)=9.8 \mathrm{~min}, \mathrm{t}_{\mathrm{R}}(S)=11.6 \mathrm{~min}\right]$.

1,4-Phenylenebis(cyanomethylene) diacetate (Derived from $4 \mathbf{p})^{7}$

${ }^{1} \mathrm{H}$ NMR (300 MHz, $\mathrm{CDCl}_{3}$ ) $\delta 2.20$ (s, 6H), 6.44 (s, 2H), 7.58-7.80. $\left.{ }^{13} \mathrm{C} \mathrm{NMR} \mathrm{(75} \mathrm{MHz,} \mathrm{CDCl}_{3}\right) \delta 20.3,62.1$, 115.5, 127.1, 129.8, 130.3, 133.0, 168.7. Chiral HPLC (AS-H; hexane/IPA = 9/1, $1.0 \mathrm{~mL} / \mathrm{min})\left[\mathrm{t}_{\mathrm{R}}(R)=60.1 \mathrm{~min}\right.$, $\left.\mathrm{t}_{\mathrm{R}}(S)=114.1 \mathrm{~min}\right]$.

\section{References}

1. I. P. Holmes, H. B. Kagan, Tetrahedron Lett. 2000, 41, 7453-7456.

2. A. Almsick, J. Buddrus, P. H. Schmidt, K. Laumen, M. P. Schneider, J. Chem. Soc., Chem. Commun. 1989, 1391-1393.

3. M. Schmidt, S. Hervé, N. Klempier, H. Griengl, Tetrahedron 1996, 52, 7833-7840. 
4. B. R. Matthews, W. R. Jackson, G. S. Jayatilake, C. Wilshire, H. A. Jacobs, Aust. J. Chem. 1988, 41, 1697-1709.

5. W.-B. Yang, J.-M. Fang, J. Org. Chem. 1998, 63, 1356-1359.

6. J.-M. Brumel, O. Legrand, G. Buono, Tetrahedron: Asymmetry 1999, 10, 1979-1984.

7. T. Dünnwald, M. Müller, J. Org. Chem. 2000, 65, 8608-8612. 


\section{Effect of water upon $(R)-1$ catalyzed enantioselective cyanation.}

Effect of water was examined with $3 a(1.0 \mathrm{mmol})$ under $10 \mathrm{~mol} \%$ of $(R)$-BINOL, $10 \mathrm{~mol} \%$ of $n$-BuLi, and $0-20 \mathrm{~mol} \%$ of $\mathrm{H}_{2} \mathrm{O}$ in toluene $(2 \mathrm{~mL})$ at $-78{ }^{\circ} \mathrm{C}$ for $1 \mathrm{~h}$. The reaction procedure was same as described in Table 1.

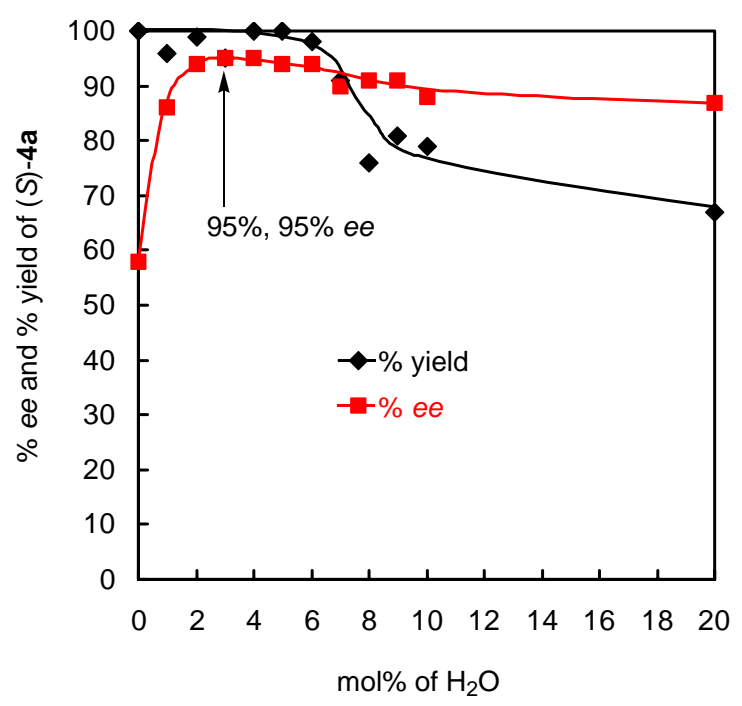

\section{Effect of water upon $(R)-2$ catalyzed enantioselective cyanation.}

Effect of water was examined with 3a $(1.0 \mathrm{mmol})$ under $10 \mathrm{~mol} \%$ of $(R)$-BINOL, $20 \mathrm{~mol} \%$ of $n$-BuLi, and 0-20 mol\% of $\mathrm{H}_{2} \mathrm{O}$ in toluene $(2 \mathrm{~mL})$ at $-78{ }^{\circ} \mathrm{C}$ for $1 \mathrm{~h}$. The reaction procedure was same as described in Table 1.

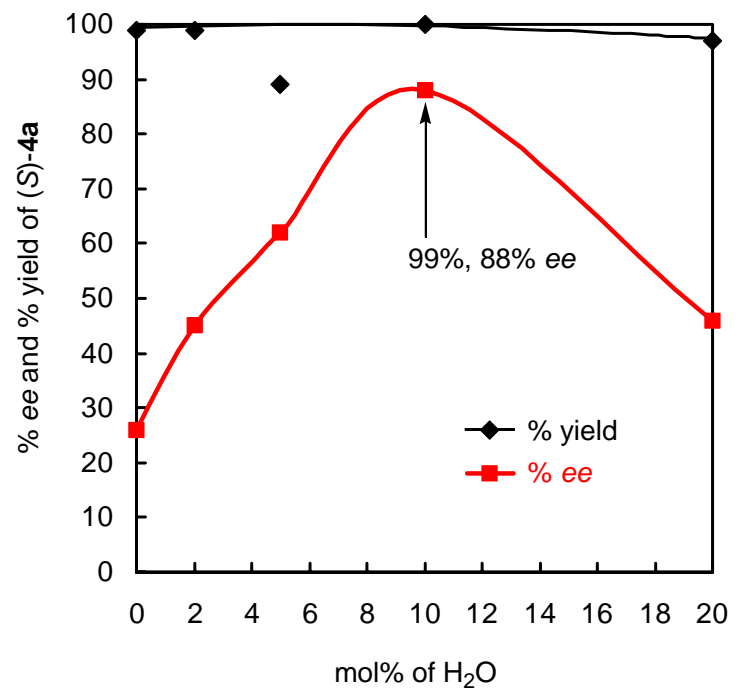


Linear effect (L.E.) between the ee of (S)-4a and the ee of (R)-BINOL [10 mol\% catalyst loading of $\left.(R)-1 \cdot\left(\mathrm{H}_{2} \mathrm{O}\right)_{n}\right]$

The L.E. was examined with $3 a(1.0 \mathrm{mmol})$ under $10 \mathrm{~mol} \%$ of $(R)$-BINOL, $10 \mathrm{~mol} \%$ of $n$-BuLi, and $3 \mathrm{~mol} \%$ of $\mathrm{H}_{2} \mathrm{O}$ in toluene $(2 \mathrm{~mL})$ at $-78{ }^{\circ} \mathrm{C}$ for $1 \mathrm{~h}$. The reaction procedure was same as described in Table 1.
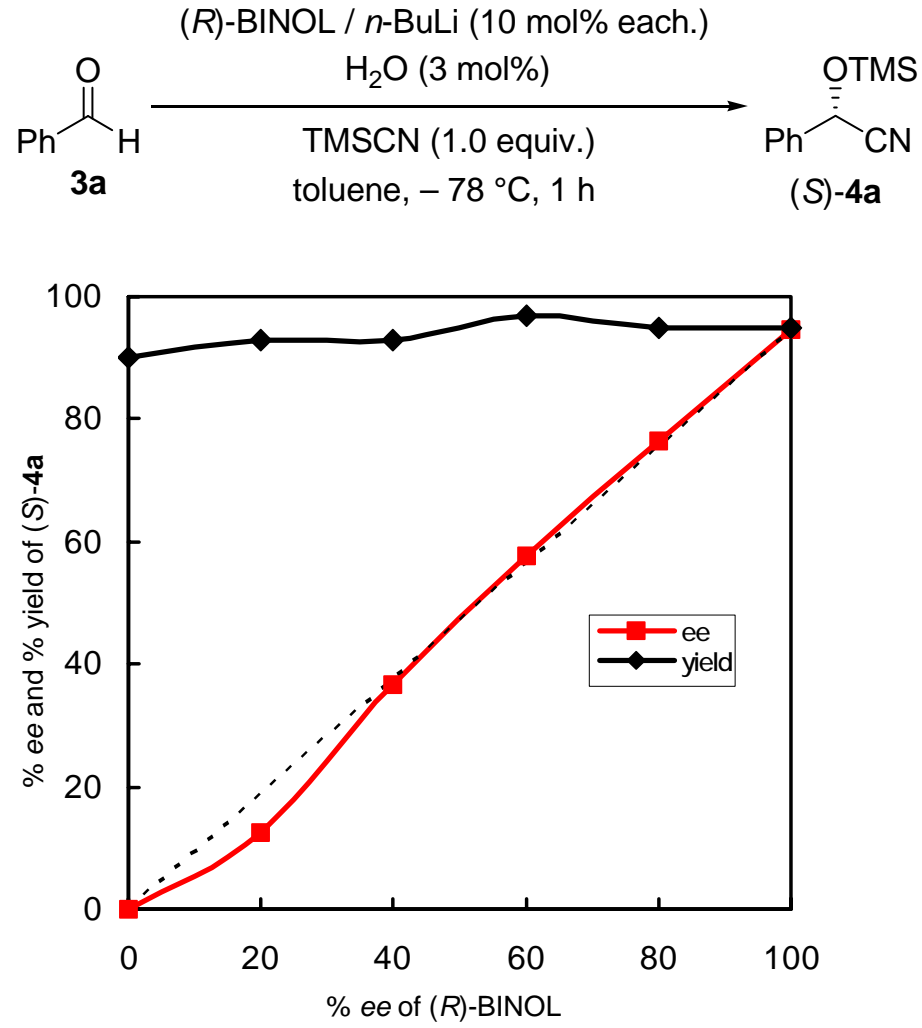
Non-linear effect (N.L.E.) between the ee of (S)-4a and the ee of (R)-BINOL [10 mol\% catalyst loading of $(R)-1 \cdot i-P r O H]$

The N.L.E. was examined with 3a (1.0 mmol) under $10 \mathrm{~mol} \%$ of $(R)$-BINOL and $10 \mathrm{~mol} \%$ of $\mathrm{LiOPr}^{i}$ in toluene $(2 \mathrm{~mL})$ at $-78{ }^{\circ} \mathrm{C}$ for $1 \mathrm{~h}$. The reaction procedure was same as described in Table 2.
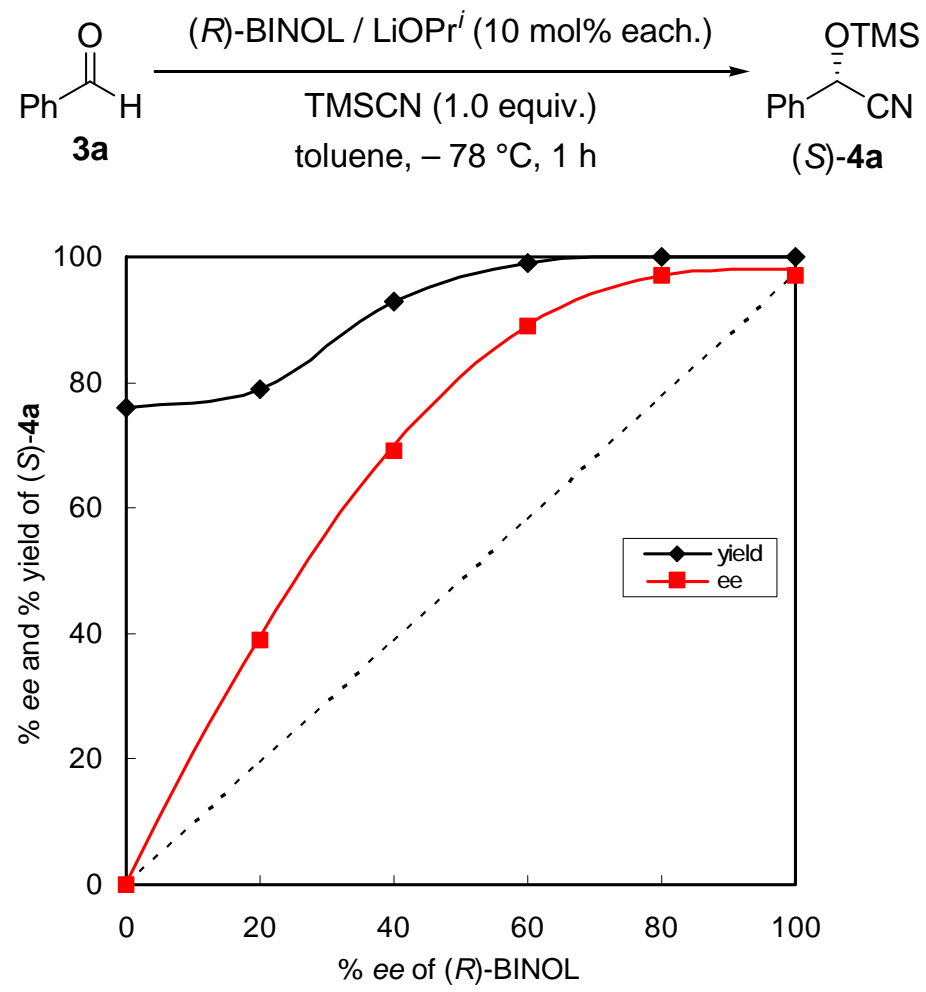
Non-linear effect (N.L.E.) between the ee of $\underline{(R)}-4 a$ and the $e e$ of $(R)$-BINOL [1 mol\% catalyst loading of 'dry' $(R)-1]$

The N.L.E. was examined under 'dry' condition with 3a (10 mmol) under 1 mol\% of (R)-BINOL and $1 \mathrm{~mol} \%$ of $n$-BuLi toluene $(2 \mathrm{~mL})$ at $-78^{\circ} \mathrm{C}$ for $1 \mathrm{~h}$. The reaction procedure was same as described in Table 1.
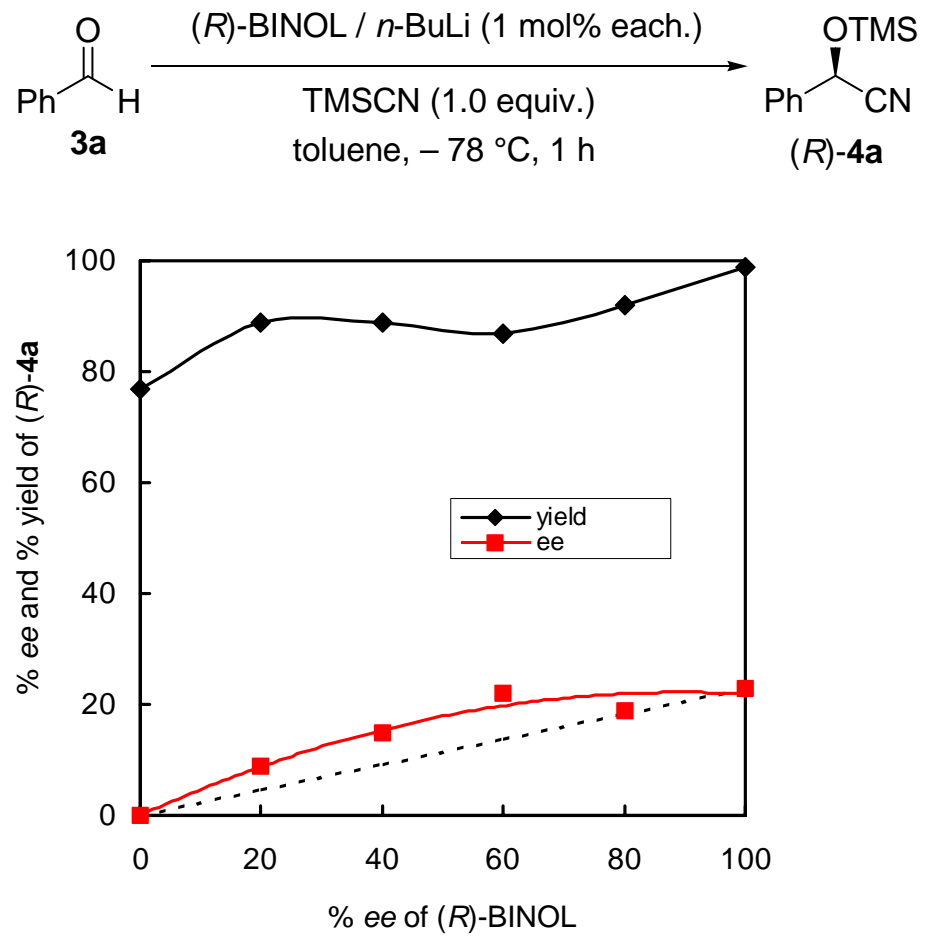
Non-linear effect (N.L.E.) between the ee of $\underline{(R)}-4 a$ and the $e e$ of $(R)$-BINOL [1 mol\% catalyst loading of 'dry' $(R)-2]$

The N.L.E. was examined under 'dry' condition with 3a (10 mmol) under 1 mol\% of (R)-BINOL and $2 \mathrm{~mol} \%$ of $n$-BuLi toluene $(2 \mathrm{~mL})$ at $-78{ }^{\circ} \mathrm{C}$ for $1 \mathrm{~h}$. The reaction procedure was same as described in Table 1.
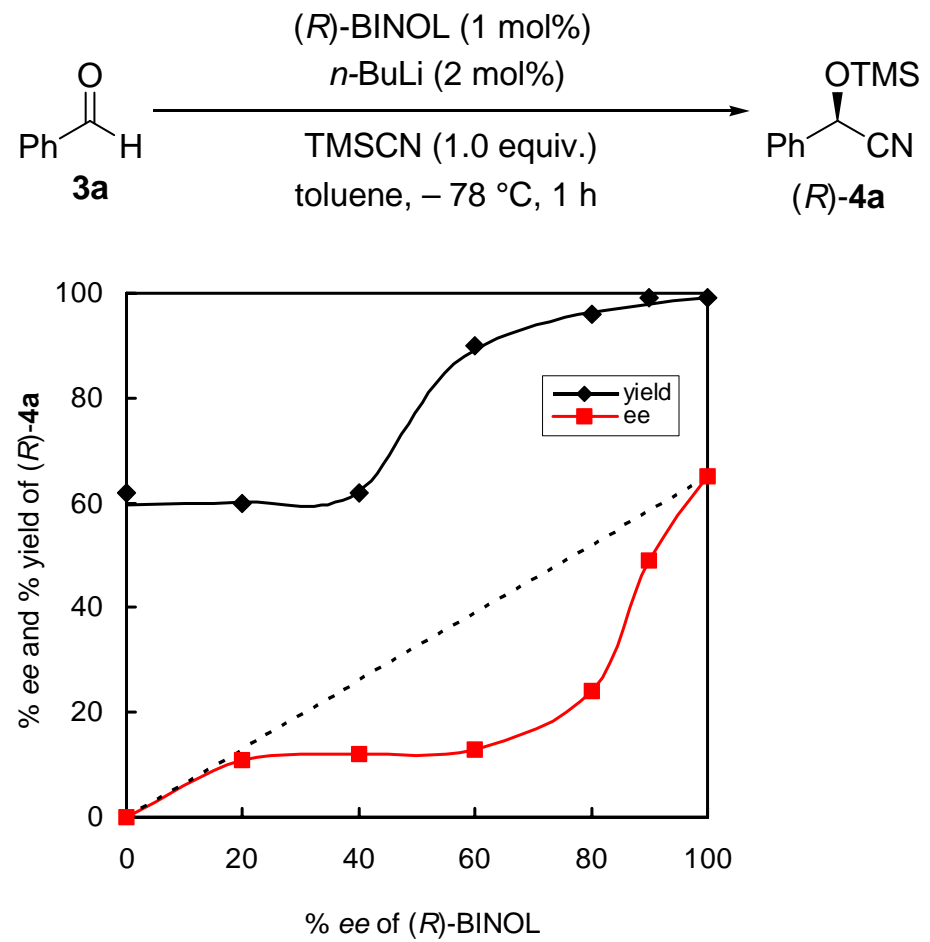
Proposed transition states (Enlarged version of Figure 1)

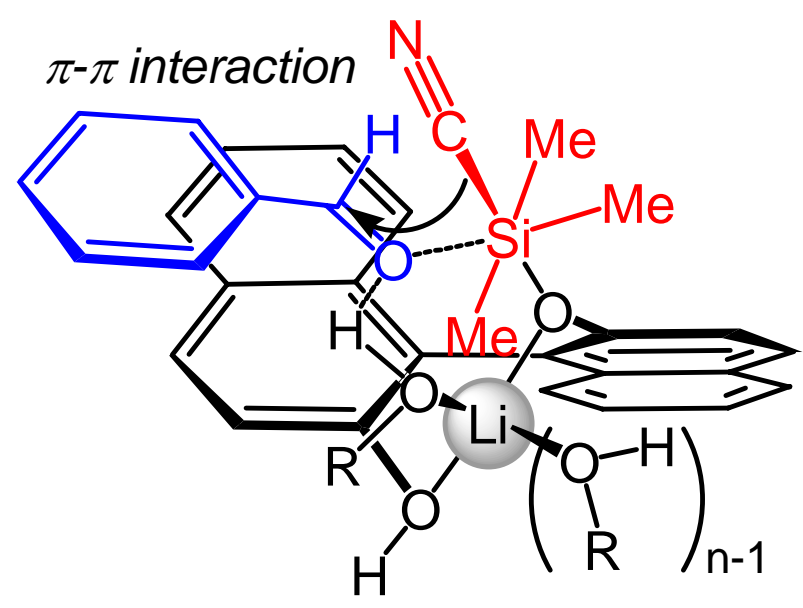

5: Favored

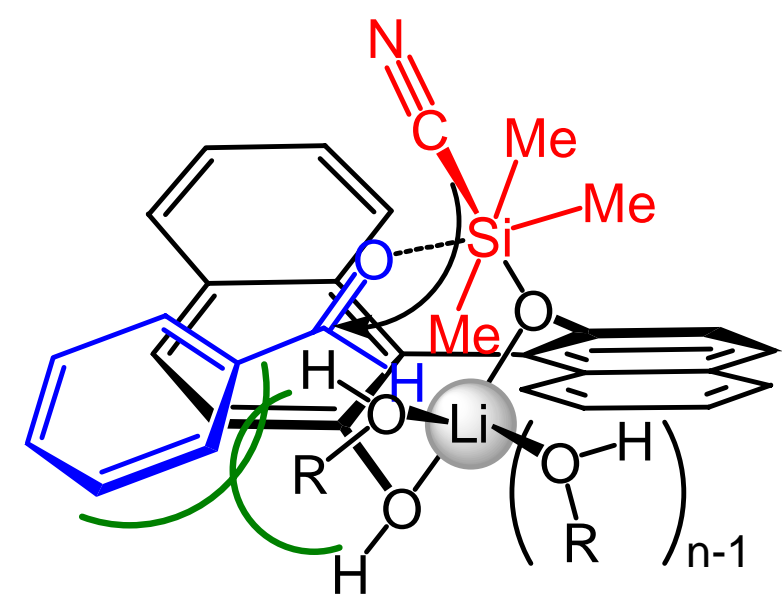

6: Disfavored 